\title{
Remembering Professor Toshikazu Oki (24 January 1935-11 April 2019)
}

\author{
Yasuhiro Igarashi ${ }^{1}$
}

Received: 9 December 2019 / Accepted: 9 December 2019 / Published online: 27 February 2020

(c) The Author(s), under exclusive licence to the Japan Antibiotics Research Association 2020

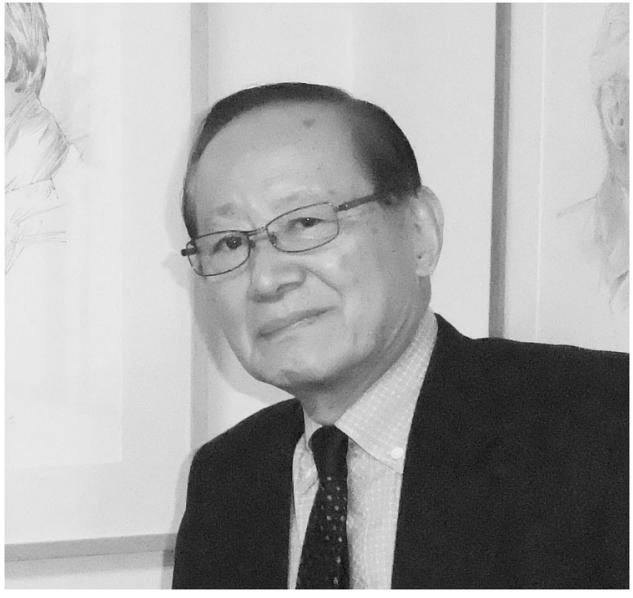

Professor Toshikazu Oki passed away on 11 April, 2019, due to a sudden onset of aspiration pneumonia after more than 60 years of antibiotic research.

Dr Oki was born in Tokyo, Japan as the first child and around the age of 10, his family moved to Hyogo where he spent his early years in the countryside. While still school in Hyogo, he met Dr Kosaku Maruyama, a biochemist who discovered the motor protein, myosin, and got inspired to pursue a career in science. After studying at Tatsuno High School in Hyogo, he was admitted to Kyoto University in 1953 where he majored in Agricultural Chemistry.

After graduation from Kyoto University, he started to work at Sanraku-Shuzou Corporation (currently, Mercian Corporation) in Yatsushiro, Kumamoto, where he was engaged in amino acid fermentation. The first scientific finding he made in the industry was the discovery of phage infection of Brevibacterium during L-glutamate fermentation,

Yasuhiro Igarashi

yas@pu-toyama.ac.jp

1 Department of Biotechnology, Toyama Prefectural University, Toyama, Japan which led to his Ph. D. thesis and the Award for Young Scientists given by Japan Society for Bioscience, Biotechnology, and Agrochemistry.

Thereafter, following changes in his company's policy to initiate cancer research, he had an opportunity to do postdoctoral research at the McArdle Laboratory for Cancer Research at the University of Wisconsin, from 1969 to 1972. At the McArdle Laboratory Dr Oki worked under the guidance of Dr Charles Heidelberger who is well known for the development of 5'-fluorouracil, an anticancer drug widely used in the clinical treatment of gastro-intestinal cancers. Having acquired wide knowledge and learned experimental techniques related to oncology in the United States, he returned to Japan when he was urged by Professor Hamao Umezawa at the Institute of Microbial Chemistry to pursue the development of anticancer drugs in Japan.

In this new role, he paid particular attention to antitumor antibiotics. By that time, adriamycin and daunomycin which exhibited potent therapeutic activity in various solid cancers had been developed in Italy. However, these drugs had serious adverse effects such as cardiac toxicity. Dr Oki noted that these antibiotics possess an anthracycline aglycone that gave a bright reddish color to the producing culture and fermentation broth. He followed up by developing a screen for microbial colonies that allowed the selection of a variety of colors and examined the antitumor activities in the extracts of these colored organisms. From this extensive work he selected a Streptomyces strain producing a yellow pigment, which was isolated and identified as a new anthracycline antibiotic named aclacinomycin (aclarubicin) with excellent antitumor activity and with milder side effects than adriamycin and daunomycin. Aclacinomycin was developed and commercialized as a new anticancer drug for blood cancers and other types of cancer in Japan. Applying the knowledge obtained from the structure and activity of aclacinomycin, he made structural modifications to adriamycin jointly with Dr Umezawa's group and Mercian group to create THP-adriamycin (Pirarubicin). These two anthracycline antibiotics have contributed greatly to 
cancer treatment and led Dr Oki to realize his dream to develop useful drugs from natural resources.

After the success at Sanraku-Ocean Corporation, Dr Oki was invited to become a director of research at Taito-Pfizer Corporation (currently, Pfizer Inc.), where he was in charge of developing and taking to market nine drugs ( $\beta$-lactams, antifungal azoles, anticancer and anti-anxiety drugs). Following these successes, in 1985, he was invited to become a managing director and research director at the Tokyo Research Center of Bristol-Myers Research Institute, Ltd. (later, Bristol-Myers Squibb Research Institute). The activity of the Tokyo Research Center was incredibly high under the leadership of Dr Oki. During this period until early 1990s, many unique bioactive compounds were discovered which led to a numerous publications by Dr Oki's research group. Personally, I was amazed at such a productive group organized by Dr Oki and at that time, I never imagined that I would work with him in my future career. The compounds discovered by Oki's group were outstanding in terms of structure and bioactivity, as exemplified by dynemicin, pradimicin, and terpestatin, all of which allured synthetic chemists worldwide to use these leads as targets for total synthesis.

In 1994, Dr Oki finally returned to academia as a professor of Biotechnology Research Center of Toyama Prefectural University, at the invitation of Professor Hideaki Yamada (Emeritus Professor of Kyoto University). This was a big challenge to Dr Oki, but he must have enjoyed the days in Toyama, because he always liked to put himself intentionally in a new environment to challenge himself in new areas of research. At the setting up of his laboratory (Laboratory of Exploratory Biotechnology Research), he invited Dr Tamotsu Furumai as an associate professor and me as an assistant professor. Dr Oki's new research group prioritized discovery of natural products from microorganisms in a diverse environment of Toyama, coupled with the collaboration with industry and academia, seeking for new bioactive molecules.
After establishing his own laboratory, Dr Oki moved back to Tokyo as a professor at Tamagawa University Research Institute in 1998 and became a specially appointed professor in 2001. During this time, he was invited to become a CEO at Translational Research Corporation from 2000 to 2002. He was a distinguished research fellow at Toray Corporate Business Research from 2006 to 2009. He was invited to become a part-time research professor at the Keck School of Medicine of the University of Southern California in 2000. He was also invited as a visiting professor at the School of Medicine of Yokohama City University in 2004. Dr Oki had consulting contracts with a number of domestic and international pharmaceutical companies. He is an inventor of more than 100 patents and an author of more than 300 scientific papers. He was the editorial board member (1986-1991) of The Journal of Antibiotics, the councilor (1992-2004) and the academic committee member (2004-2019) of Japan Antibiotics Research Association. In 2001 he received SAJ Merit Award from The Society for Actinomycetes Japan for his great contribution to the research on actinomycete antibiotics.

Dr Oki's favorite word was 'serendipity', by which his great achievements are literally symbolized but his mind was always searching for the serendipity. He was not merely a hard worker but also a heartful mentor and a family-oriented man. His death was a great loss to the natural products and antibiotic community, as well as for his family and friends. After he lost his beloved wife Reiko, he was in deep grief, and in recent years, he spent peaceful days and enjoyed staying with his children and grandchildren.

Dr Oki left a personal autobiography to his family when he was 80 years old. Mrs. Reiko was still alive at that time. In its closing remarks, he wrote "My wish is to watch the 2020 Olympic-Paralympic Games in Tokyo with my wife on TV....". In this sorrowful time, all of his friends and colleagues extend their deepest sympathies to Dr Oki's family. 